\title{
Predictive Analysis for the Detection of Cardio Vascular Disease (CVD) based on Machine Learning Classification Algorithm
}

\author{
Dillip Narayan Sahu ${ }^{1}$, Vijay Pal Singh ${ }^{2 *}$ \\ ${ }^{1}$ Research Scholar, Department of Computer Science, OPJS University, Rajasthan, India \\ ${ }^{2}$ Associate Professor, Department of Computer Science, OPJS University, Rajasthan, India
}

\begin{abstract}
Cardio Vascular Disease (CVD) is a leading cause of death worldwide. It is also called heart disease. As per the WHO report, around 17.9 million people die every year from Cardio Vascular Diseases, an estimated 31\% of all deaths globally, and patients die mainly because of non-appropriate and un-affordable treatment. Heart related diseases are a worldwide major health crisis in the present scenario. This disease can be curable with early diagnosis and proper treatment[1][2]. The purpose of this paper is to establish some predictive analytical models using Machine Learning algorithms by taking a real time CVD dataset. In this paper, we have shown some experimental observations with the help of some Machine Learning classification algorithms, and also shown a clear vision on the predictive analysis on medical diagnosis of the Cardio Vascular Disease(CVD) using Machine Learning algorithms using which patients may get benefited by the accurate result for the better diagnose in their early treatment.
\end{abstract}

Keywords: Algorithm, Cardio Vascular Disease, Classifier, Machine Learning, Predictive Analysis.

\section{INTRODUCTION}

According to description asub-umbrella term for a number of conditions that affect the heart itself and/ or the blood vessel system. Especially the modes and highways leading to and from the heart[3][4].

Causes of cardiovascular complaint include diabetes mellitus, hypertension and hyperactive cholesterolemia.

Symptoms

The main symptoms of cardiovascular disease are:

Chest pain, Shortness of breath Pain in the body, Feeling faint, Feeling sick

Diagnosis through Machine Learning

- Electrocardiogram (ECG) - Nuclear stress testing

- Echocardiogram (ECHO) - Coronary angiogram

- CT scan

- PET/CT scan

- Magnetic Resonance Imaging (MRI)

Risk Factor

- Age - Absence of key nutritional elements such as polyphonic antioxidants

- Diabetes mellitus - Hyper cholesterolemia

- Tobacco smoking • Higher fibrinogen and PAI-1 blood concentrations

Prevention

- Smoking conclusion (or abstinence) is one of the most effective and fluently adjustable changes

- Regular cardiovascular exercise complements the healthful eating habits Treatment through Machine Learning [5]

- Implantable cardioverter-defibrillator: controlling a chaotic heart

- Pacemakers: Generating regular heartbeats: Angiotensin-II receptor blockers.

Experiments and Observations-1

We have taken Weka-Wekato Environment for Knowledge Analysis Machine Learning tool to preprocess, clean, data prediction and classification of the CVD (Heart) disease based on the real-time Cardio Vascular Disease patient dataset(in .CSV and Attribute Relation File Format .arff)[6]. 


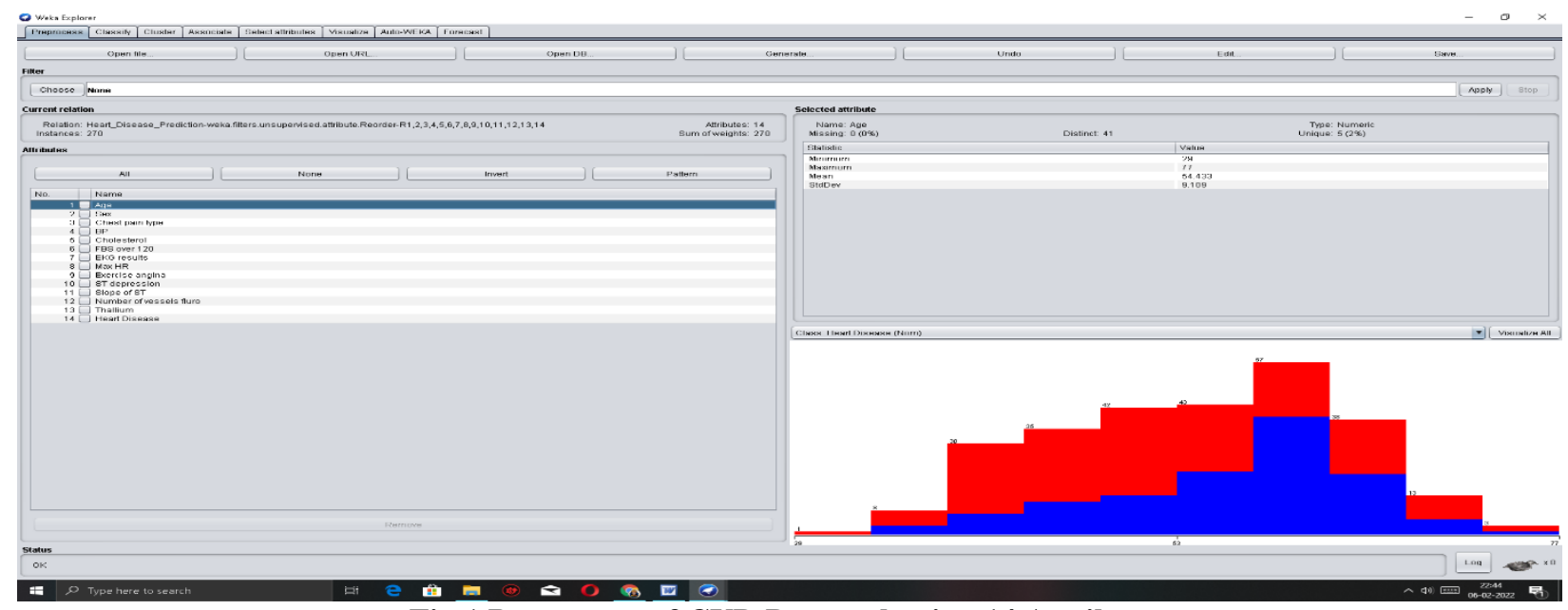

Fig.1 Preprocess of CVD Dataset having 14 Attributes

Bagging- Class for bagging a classifier to reduce variance.

\section{Classifier Output}

$===$ Run information $===$

Scheme: weka.classifiers.meta.Bagging -P 100 -S 1 -num-slots 1 -I 10 -W weka.classifiers.trees.REPTree -- -M 2 -V 0.001 -N 3 -S 1 -L - 1 -I 0.0

Relation: Heart_Disease_Prediction-weka.filters.unsupervised.attribute.Reorder-R1,2,3,4,5,6,7,8,9,10,11,12,13,14weka.filters.AllFilter-weka.filters.MultiFilter-Fweka.filters.AllFilter

Instances: $270 \quad$ Attributes: 14

Age Sex Chest pain type BP Cholesterol FBS over 120 EKG results Max HR Exercise angina $\quad$ ST depression $\quad$ Slope of ST Number of vessels fluro

\section{Thallium Heart Disease}

Test mode: 10-fold cross-validation

$===$ Classifier model (full training set) $===$

Bagging with 10 iterations and base learner

weka.classifiers.trees.REPTree -M 2 -V 0.001 -N 3 -S 1 -L -1 -I 0.0

Time taken to build model: 0.05 seconds

$===$ Stratified cross-validation $======$ Summary $===$

Correctly Classified Instances $242 \quad \mathbf{8 9 . 6 2} \%$

Incorrectly Classified Instances $28 \quad 10.38 \%$

Experiments and Observations-2

Stacking- Combines several classifiers using the stacking method.

Classifier Output

$===$ Run information $===$

Scheme: $\quad$ weka.classifiers.meta.Stacking $-\mathrm{X} \quad 10$-M "weka.classifiers.rules.ZeroR " $\quad$-S 1 -num-slots 1 -B "weka.classifiers.rules.ZeroR "

Relation: Heart_Disease_Prediction-weka.filters.unsupervised.attribute.Reorder-R1,2,3,4,5,6,7,8,9,10,11,12,13,14weka.filters.AllFilter-weka.filters.MultiFilter-Fweka.filters.AllFilter

Instances: $270 \quad$ Attributes: 14

Test mode: 10 -fold cross-validation

$===$ Classifier model (full training set) $===$

Stacking

Base classifiers

ZeroR predicts class value: Absence

Meta classifier

ZeroR predicts class value: Absence

Time taken to build model: 0 seconds

$===$ Stratified cross-validation $======$ Summary $===$

Correctly Classified Instances $\quad 150 \quad 55.5556 \%$

Incorrectly Classified Instances $120 \quad 44.4444 \%$

Kappa statistic

Mean absolute error

0 
Root mean squared error

Relative absolute error

0.4969
$100 \%$
$100 \%$
270

Total Number of Instances

$===$ Detailed Accuracy By Class $===$

TP Rate FP Rate Precision Recall F-Measure MCC ROC Area PRC Area Class
$0.000 \quad 0.000 \quad ?$
0.000
$? \quad 0.500 \quad 0.444 \quad$ Presence
$\begin{array}{llllllllll}1.000 & 1.000 & 0.556 & 1.000 & 0.714 & ? & 0.500 & 0.556 & \text { Absence }\end{array}$

$\begin{array}{lllllllll}\text { Weighted Avg. } & 0.556 & 0.556 & ? & 0.556 & ? & ? & 0.500 & 0.506\end{array}$

$===$ Confusion Matrix $===$

a $b<--$ classified as

$0120 \mid \mathrm{a}=$ Presence

$0150 \mid \mathrm{b}=$ Absence

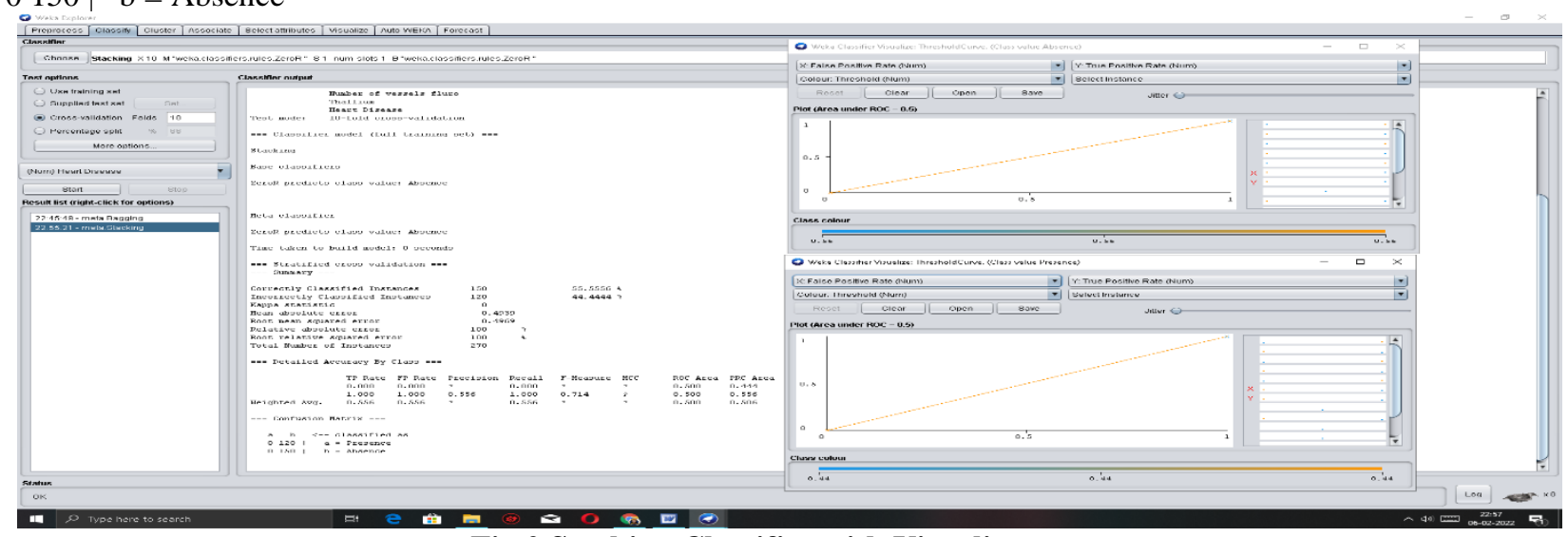

Fig.2 Stacking Classifier with Visualize curve

Experiments and Observations-3

UltraBoost- UltraBoost adaptively boosts (AdaBoosts) heterogeneous classifiers: a different classifier can be boosted at each stage.

\section{Classifier Output}

$===$ Run information $===$

Scheme:

weka.classifiers.meta.UltraBoost Relation:

Heart_Disease_Prediction-

weka.filters.unsupervised.attribute.Reorder-R1,2,3,4,5,6,7,8,9,10,11,12,13,14-weka.filters.AllFilter-

weka.filters.MultiFilter-Fweka.filters.AllFilter

Instances: $270 \quad$ Attributes: 14

Test mode: 10 -fold cross-validation

$===$ Classifier model (full training set $)===$

UltraBoost

Base classifiers

FilteredClassifier using weka.classifiers.bayes.NaiveBayes on data filtered through weka.filters.unsupervised.attribute.RemoveType $-\mathrm{V}-\mathrm{T}$ nominal

Filtered Header

@ relation_'Heart_Disease_Prediction-weka.filters.unsupervised.attribute.Reorder-R1,2,3,4,5,6,7,8,9,10,11,12,13,14weka.filters.AllFilter-weka.filters.MultiFilter-Fweka.filters.AllFilter-weka.filters.unsupervised.attribute.RemoveType-

V-Tnominal'

@attribute 'Heart Disease' \{Presence,Absence\}

@ data

Classifier Model

Naive Bayes Classifier

Class

Attribute Presence Absence

(0.45) (0.55)

FilteredClassifier using weka.classifiers.functions.Logistic $-\mathrm{R} 1.0 \mathrm{E}-8$-M -1 -num-decimal-places 4 on data filtered through weka.filters.unsupervised.attribute.RemoveType $-\mathrm{V}-\mathrm{T}$ numeric

Filtered Header 
@ relation_Heart_Disease_Prediction-weka.filters.unsupervised.attribute.Reorder-R1,2,3,4,5,6,7,8,9,10,11,12,13,14weka.filters.AllFilter-weka.filters.MultiFilter-Fweka.filters.AllFilter-weka.filters.unsupervised.attribute.RemoveTypeV-Tnumeric'

Classifier Model

Logistic Regression with ridge parameter of $1.0 \mathrm{E}-8$

Coefficients...

$\begin{array}{lc}\text { Variable } & \begin{array}{c}\text { Class } \\ \text { Presence }\end{array} \\ \text { ============================ } & -0.0169 \\ \text { Age } & 1.5578 \\ \text { Sex } & 0.6981 \\ \text { Chest pain type } & 0.0253 \\ \text { BP } & 0.0073 \\ \text { Cholesterol } & -0.8046 \\ \text { FBS over 120 } & 0.3002 \\ \text { EKG results } & -0.0212 \\ \text { Max HR } & 0.8217 \\ \text { Exercise angina } & 0.3418 \\ \text { ST depression } & 0.4437 \\ \text { Slope of ST } & 0.3412 \\ \text { Number of vessels fluro } 1.168 \\ \text { Thallium } & -8.4283 \\ \text { Intercept } & \text { Class } \\ \text { Odds Ratios... } & \text { Presence } \\ \text { Variable } & \end{array}$

\begin{tabular}{|c|c|c|c|}
\hline Age & \multicolumn{3}{|l|}{0.9832} \\
\hline Sex & \multicolumn{3}{|l|}{4.7483} \\
\hline Chest pain type & \multicolumn{3}{|l|}{2.0099} \\
\hline $\mathrm{BP}$ & \multicolumn{3}{|l|}{1.0256} \\
\hline Cholesterol & \multicolumn{3}{|l|}{1.0074} \\
\hline FBS over 120 & \multicolumn{3}{|l|}{0.4473} \\
\hline EKG results & \multicolumn{3}{|l|}{1.3501} \\
\hline Max HR & \multicolumn{3}{|l|}{0.979} \\
\hline Exercise angina & \multicolumn{3}{|l|}{2.2743} \\
\hline ST depression & \multicolumn{3}{|l|}{1.4075} \\
\hline Slope of ST & \multicolumn{3}{|l|}{1.5584} \\
\hline \multicolumn{4}{|c|}{ Number of vessels fluro $\quad 3.2156$} \\
\hline Thallium & \multicolumn{3}{|l|}{1.4066} \\
\hline \multicolumn{4}{|c|}{$===$ Stratified cross-validation $======$ Summary $===$} \\
\hline Correctly Classi & d Instances & 225 & $83.3333 \%$ \\
\hline Incorrectly Clas & ied Instances & 45 & $16.6667 \%$ \\
\hline Kappa statistic & \multicolumn{3}{|c|}{0.6599} \\
\hline Mean absolute e & \multicolumn{3}{|c|}{0.3364} \\
\hline Root mean squas & \multicolumn{3}{|c|}{0.3723} \\
\hline Relative absolut & \multicolumn{3}{|c|}{$68.1079 \%$} \\
\hline Root relative squ & \multicolumn{3}{|c|}{$74.9264 \%$} \\
\hline Total Number of & nstances & \multicolumn{2}{|l|}{270} \\
\hline
\end{tabular}

$===$ Detailed Accuracy By Class ===

TP Rate FP Rate Precision Recall F-Measure MCC ROC Area PRC Area Class

$\begin{array}{lllllllll}0.775 & 0.120 & 0.838 & 0.775 & 0.805 & 0.661 & 0.903 & 0.884 & \text { Presence } \\ 0.880 & 0.225 & 0.830 & 0.880 & 0.854 & 0.661 & 0.903 & 0.919 & \text { Absence } \\ \text { Avg. } & 0.833 & 0.178 & 0.834 & 0.833 & 0.833 & 0.661 & 0.903 & 0.903\end{array}$

$\begin{array}{lllllllll}\text { Weighted Avg. } & 0.833 & 0.178 & 0.834 & 0.833 & 0.833 & 0.661 & 0.903 & 0.903\end{array}$

\footnotetext{
$===$ Confusion Matrix $===$

a $b$ <-- classified as

$9327 \mid \mathrm{a}=$ Presence
} 
$18132 \mid \mathrm{b}=$ Absence

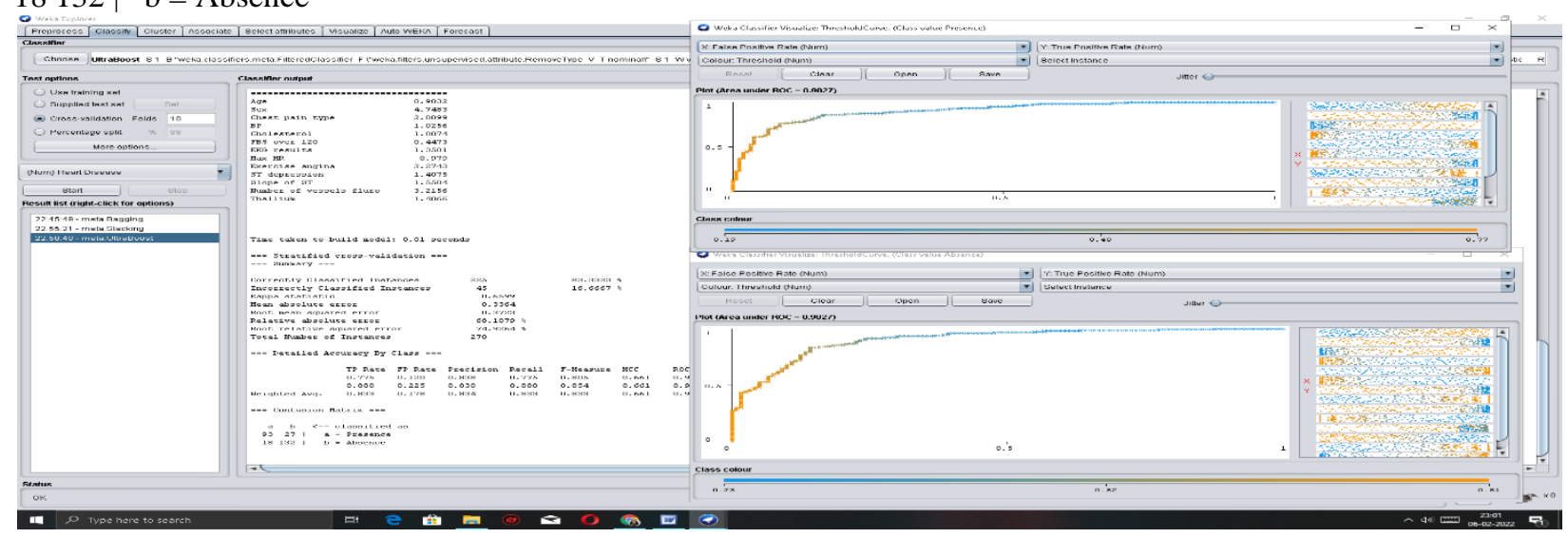

Fig.3 UltraBoost Classifier with Visualize curve

\section{Experiments and Observations-4}

Vote- Class for combining classifiers..

\section{Classifier Output}

$===$ Run information $===$

Scheme: weka.classifiers.meta.Vote -S 1 -B "weka.classifiers.rules.ZeroR " -R AVG

Relation: Heart_Disease_Prediction-weka.filters.unsupervised.attribute.Reorder-R1,2,3,4,5,6,7,8,9,10,11,12,13,14weka.filters.AllFilter-weka.filters.MultiFilter-Fweka.filters.AllFilter

Instances: $270 \quad$ Attributes: $14 \quad$ Test mode: 10-fold cross-validation

$===$ Classifier model (full training set) $===$

Vote combines the probability distributions of these base learners:

weka.classifiers.rules.ZeroR using the 'Average' combination rule

All the models:

ZeroR predicts class value: Absence

Time taken to build model: 0 seconds

$===$ Detailed Accuracy By Class ===

TP Rate FP Rate Precision Recall F-Measure MCC ROC Area PRC Area Class

$\begin{array}{ccccccccccc}0.000 & 0.000 & ? & 0.000 & ? & ? & 0.500 & 0.444 & \text { Presence } \\ 1.000 & 1.000 & 0.556 & 1.000 & 0.714 & ? & 0.500 & 0.556 \quad \text { Absence } \\ \text { Avg. } & 0.556 & 0.556 & ? & 0.556 & ? & & ? & 0.500 & 0.506\end{array}$

$\begin{array}{llllllll}\text { Weighted Avg. } & 0.556 & 0.556 & ? & 0.556 & ?\end{array}$

$===$ Confusion Matrix $===$

a $b \quad<--$ classified as

$0120 \mid \mathrm{a}=$ Presence

$0150 \mid \mathrm{b}=$ Absence

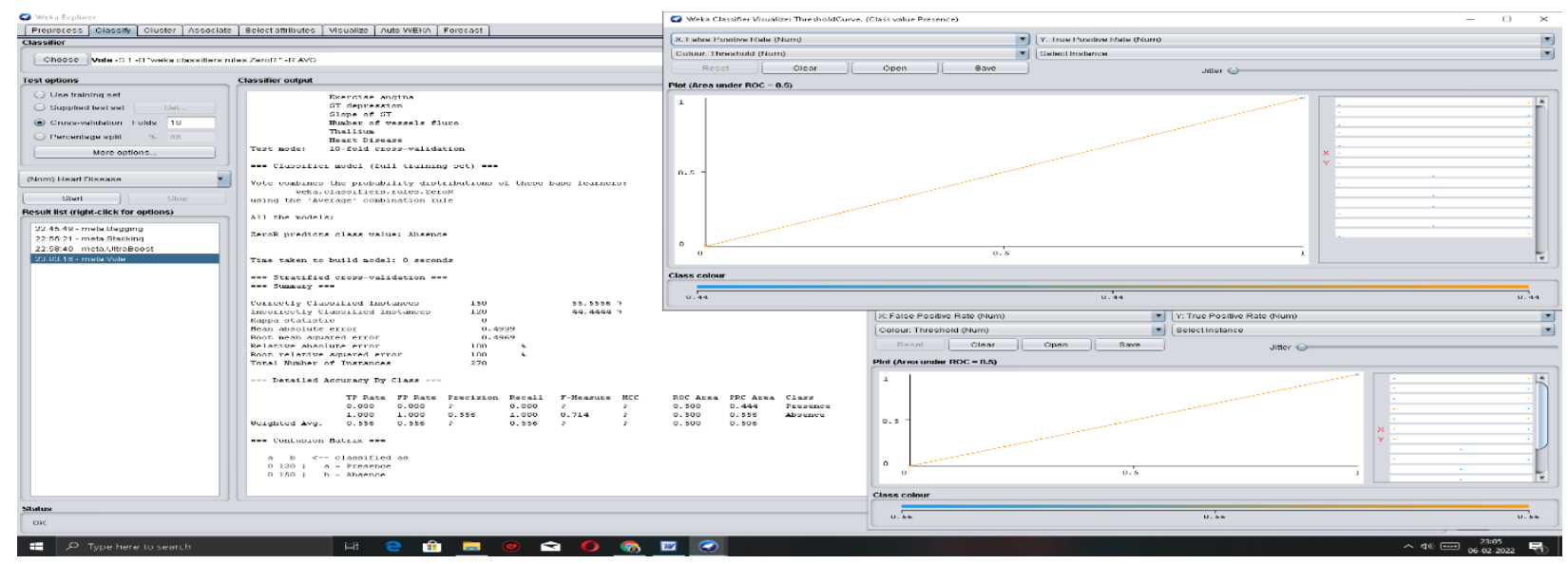

Fig.4 Vote Classifier with Visualize curve 
Experiments and Observations-5

NaiveBayes- Class for a Naive Bayes classifier using estimator classes.

Classifier Output

$===$ Run information $===$

Scheme: weka.classifiers.bayes.NaiveBayes

Relation: Heart_Disease_Prediction-weka.filters.unsupervised.attribute.Reorder-R1,2,3,4,5,6,7,8,9,10,11,12,13,14weka.filters.AllFilter-weka.filters.MultiFilter-Fweka.filters.AllFilter

Instances: $270 \quad$ Attributes: 14

Test mode: 10-fold cross-validation

$===$ Classifier model (full training set) $===$

Naive Bayes Classifier Class

Attribute Presence Absence

$$
\text { (0.44) (0.56) }
$$

$===$ Stratified cross-validation $======$ Summary $===$

$\begin{array}{lcc}\text { Correctly Classified Instances } & 228 & 84.4444 \% \\ \text { Incorrectly Classified Instances } & 42 & 15.5556 \% \\ \text { Kappa statistic } & 0.6834 & \\ \text { Mean absolute error } & 0.1814 & \\ \text { Root mean squared error } & 0.356 & \\ \text { Relative absolute error } & 36.7302 \% & \\ \text { Root relative squared error } & 71.6335 \% & \\ \text { Total Number of Instances } & 270 & \end{array}$

=== Detailed Accuracy By Class ===

TP Rate FP Rate Precision Recall F-Measure MCC ROC Area PRC Area Class

$\begin{array}{lllllllll}0.800 & 0.120 & 0.842 & 0.800 & 0.821 & 0.684 & 0.901 & 0.875 & \text { Presence }\end{array}$

$\begin{array}{lllllllll}0.880 & 0.200 & 0.846 & 0.880 & 0.863 & 0.684 & 0.901 & 0.917 & \text { Absence }\end{array}$

$\begin{array}{lllllllll}\text { Weighted Avg. } & 0.844 & 0.164 & 0.844 & 0.844 & 0.844 & 0.684 & 0.901 & 0.898\end{array}$

$===$ Confusion Matrix ===

a $b$ <-- classified as

$9624 \mid \mathrm{a}=$ Presence

$18132 \mid \mathrm{b}=$ Absence

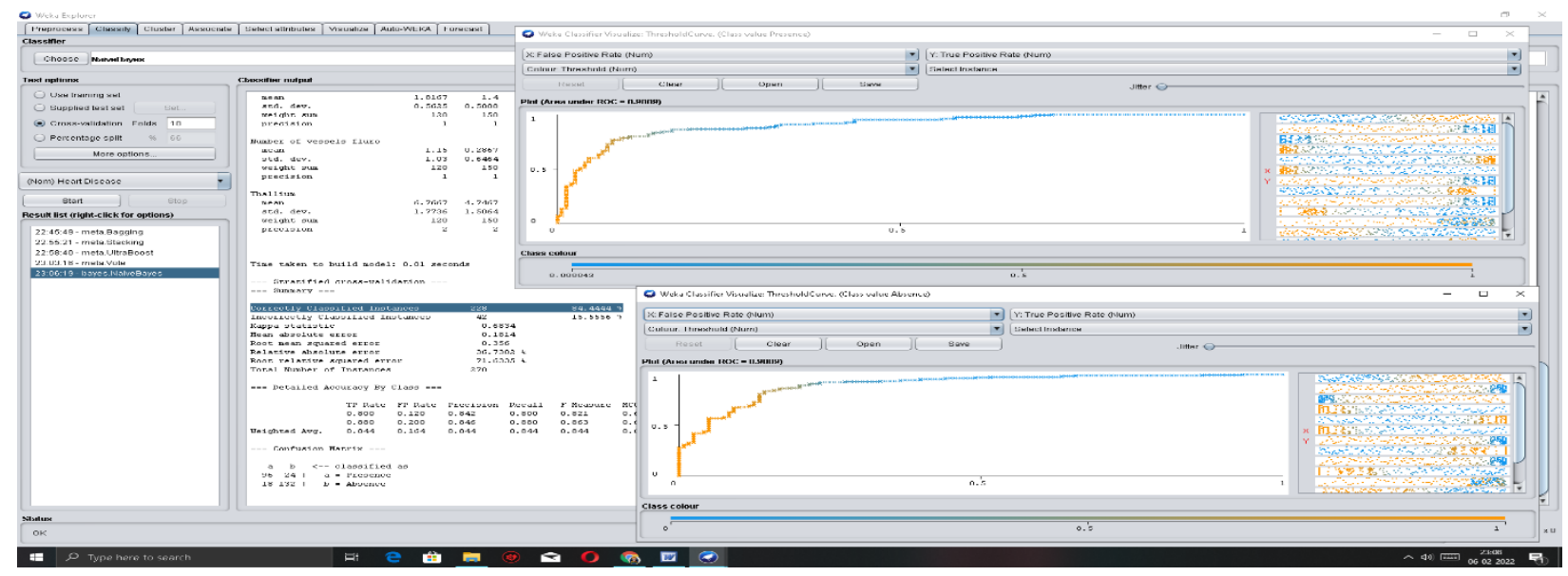

Fig.5 NaiveBayes Classifier with Visualize curve 


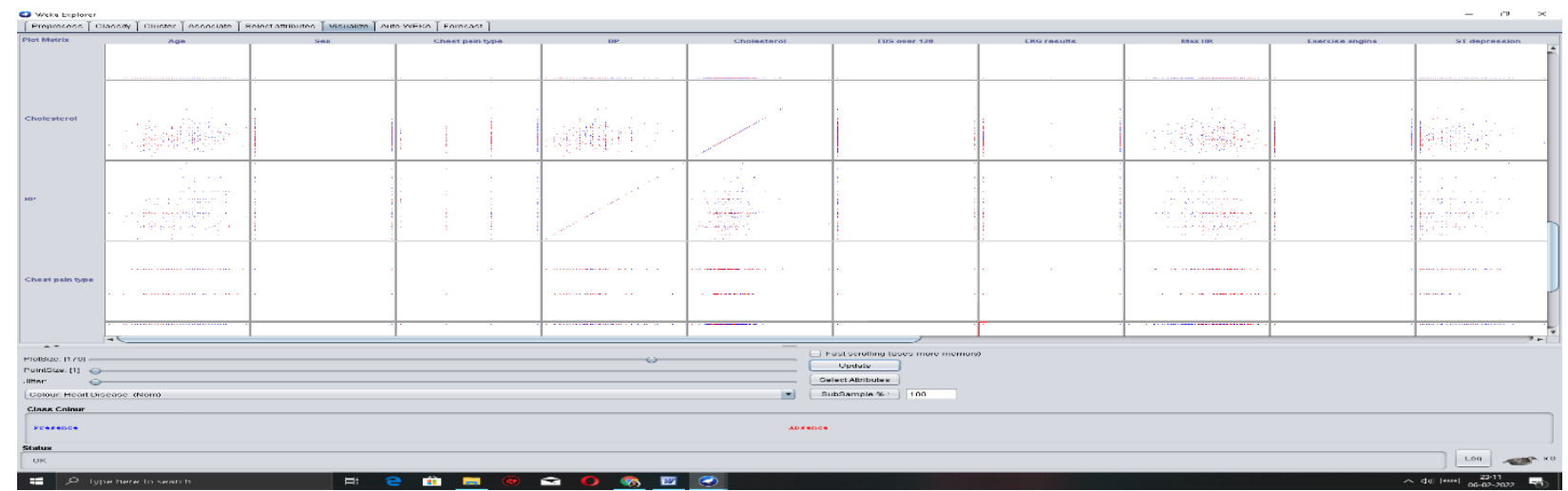

Fig.6 Visualize Plot Matrix for all Attributes

\section{CONCLUSION}

We have taken 5 different machine learning classifier algorithms and with the observations to decide the acceptability of a particular domain in the machine learning model. In the study of the above real time medical dataset implementation and in different observations, it is found that the accuracy level using the machine learning classification model Bagging is very much satisfactory, having good accuracy rate of $89.62 \%$ and so will be a good option in the field of medical sciences to predict early diagnosis of Cardio Vascular Disease. As we have taken 5 different experimental observations using the machine learning tool to clearly analyze, detect and predict for the Cardio Vascular Disease. In the study of the above experimental observations, it is found that, machine learning tools are no doubt an excellent way to predict and detect the Cardio Vascular disease (Heart) at an early stage prior to the satisfiability of the conditions of the early stage patient. It is found that the accuracy level using different algorithm in Machine Learning is an excellent option for detection and prediction of Cardio Vascular disease, having good accuracy rate and so will be efficient and acceptable.

\section{REFERENCES}

[1] Jha, V., Garcia-Garcia, G., Iseki, K., Li, Z., Naicker, S., Plattner, B. \& Yang, C. W. (2013). Chronic kidney disease: global dimension and perspectives. The Lancet, 382(9888), 260-272.

[2] Ali, S., Dave, N., Virani, S. S., \& Navaneethan, S. D. (2019). Primary and secondary prevention of cardiovascular disease in patients with chronic kidney disease. Current Atherosclerosis Reports, 21(9), 1-9.

[3] Levey, A. S., Coresh, J., Bolton, K., Culleton, B., Harvey, K. S., Ikizler, T. A. \& Briggs, J. (2002). K/DOQI clinical practice guidelines for chronic kidney disease: evaluation, classification, and stratification. American Journal of Kidney Diseases, 39(2 SUPPL. 1), i-ii+.

[4] Jordan, M. I., \& Mitchell, T. M. (2015). Machine learning: Trends, perspectives, and prospects. Science, 349(6245), 255-260.

[5] Gunarathne, W. H. S. D., Perera, K. D. M., \& Kahandawaarachchi, K. A. D. C. P. (2017, October). Performance evaluation on machine learning classification techniques for disease classification and forecasting through data analytics for chronic kidney disease (CKD). In 2017 IEEE 17th International Conference on Bioinformatics and Bioengineering (BIBE) (pp. 291-296). IEEE.

[6] Chen, T., \& Guestrin, C. (2016, August). Xgboost: A scalable tree boosting system. In Proceedings of the 22nd ACM Sigkdd International Conference on Knowledge Discovery and Data Mining (pp. 785-794).

[7] Noble, W. S. (2006). What is a support vector machine?. Nature Biotechnology, 24(12), 1565-1567. [8] Pregibon, D. (1981). Logistic regression diagnostics. Annals of Statistics, 9(4), 705-724.

[9] Lakshmi, K. R., Nagesh, Y., \& Krishna, M. V. (2014). Performance comparison of three data mining techniques for predicting kidney dialysis survivability. International Journal of Advances in Engineering \& Technology, 7(1), 242.

[10] Vijayarani, S., Dhayanand, S., \& Phil, M. (2015). Kidney disease prediction using SVM and ANN algorithms. International Journal of Computing and Business Research (IJCBR), 6(2), 1-12. [11] Baby, P. S., \& Vital, T. P. (2015). Statistical analysis and predicting kidney diseases using machine learning algorithms. International Journal of Engineering Research and Technology, 4(7), 206-210. 